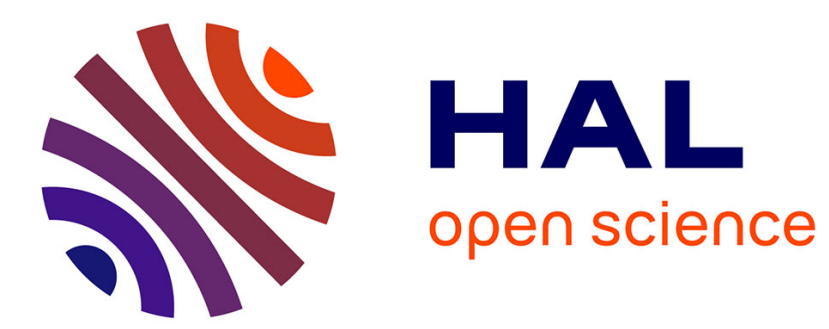

\title{
How good are SPT schedules for fair optimality criteria
}

Eric Angel, Evripidis Bampis, Fanny Pascual

\section{To cite this version:}

Eric Angel, Evripidis Bampis, Fanny Pascual. How good are SPT schedules for fair optimality criteria. Annals of Operations Research, 2008, 159 (1), pp.53-64. 10.1007/s10479-007-0267-0 . hal-00341351

\section{HAL Id: hal-00341351 \\ https://hal.science/hal-00341351}

Submitted on 19 Jul 2009

HAL is a multi-disciplinary open access archive for the deposit and dissemination of scientific research documents, whether they are published or not. The documents may come from teaching and research institutions in France or abroad, or from public or private research centers.
L'archive ouverte pluridisciplinaire HAL, est destinée au dépôt et à la diffusion de documents scientifiques de niveau recherche, publiés ou non, émanant des établissements d'enseignement et de recherche français ou étrangers, des laboratoires publics ou privés. 


\title{
HOW GOOD ARE SPT SCHEDULES FOR FAIR OPTIMALITY CRITERIA
}

\author{
Eric Angel, ${ }^{1}$ Evripidis Bampis, ${ }^{1}$ Fanny Pascual ${ }^{1}$ \\ ${ }^{1}$ LaMI, CNRS UMR 8042, Université d'Evry, Tour Evry 2, 523, Place des Terrasses 91000 \\ Evry, France \\ \{angel, bampis, fpascual\}@lami.univ-evry.fr
}

\begin{abstract}
We consider the following scheduling setting: a set of $n$ tasks have to be executed on a set of $m$ identical machines. It is well known that shortest processing time (SPT) schedules are optimal for the problem of minimizing the total sum of completion times of the tasks. In this paper, we measure the quality of SPT schedules, from an approximation point of view, with respect to the following optimality criteria: sum of completion times per machine, global fairness, and individual fairness.
\end{abstract}

\section{Introduction}

We are given a set of $n$ tasks, $T_{1}, T_{2}, \cdots, T_{n}$, with execution times $l_{1}, l_{2}, \cdots$, $l_{n}$ and a set of $m$ identical machines (or processors) $P_{1}, P_{2}, \cdots, P_{m}$. Given a schedule we denote by $C_{j}$ the completion time of task $T_{j}$, and by $X$ (resp. $\vec{X}$ ) the completion time vector whose $i^{\text {th }}$ coordinate is the completion time of task $T_{i}$ (resp. where the coordinates of $X$ have been reordered in non increasing order). For each instance $\mathcal{I}$ we define $V(\mathcal{I})$ to be the set of all vectors that are induced by some feasible schedule. One of the most popular optimality criteria in scheduling theory is the sum of completion times criterion defined as $\sum_{i=1}^{n} C_{i}$. An optimal solution to the problem of minimizing the sum of completion times can be constructed by using the shortest execution time (or Smith's) rule [1]: sort the tasks in non decreasing processing time order and schedule the tasks greedily on the machines following this order: when a machine becomes idle it executes the smallest unscheduled task. A broader class of optimal schedules for the sum of completion times criterion is the following class of schedules that are defined using the notion of rank [1]. Roughly speaking, a task is at the $t^{\text {th }}$ rank if its execution time is smaller than or equal to the execution times of the tasks at a largest rank, and largest than or equal to the execution times of the tasks scheduled at a smallest rank. 
More formally, we denote by $\xi$ the ordered collection $\left(l_{1}, \ldots, l_{n}\right)$ and we assume that the indexing is chosen so that $l_{1} \leq l_{2} \leq \cdots \leq l_{n}$. Our scheduling instance $\mathcal{I}$ is completely defined by the system $(\xi, m)$, where $m$ is the number of (identical) processors. Let $\pi_{i}$ be a collection of processing times defined by $\pi_{k}=\left\{l_{n}, l_{n-1}, \ldots, l_{n-m+1}\right\}, \pi_{k-1}=\left\{l_{n-m}, \ldots, l_{n-2 m+1}\right\}, \ldots$, $\pi_{1}=\left\{l_{n-(k-1) m}, \ldots, l_{1}\right\}$, where $k=\left\lceil\frac{n}{m}\right\rceil$. The collection $\pi_{i}$ is called the $i^{\text {th }}$ rank of tasks with respect to $(\xi, m)$. Consider a schedule obtained by scheduling the tasks rank-by-rank in the order $\pi_{1}, \pi_{2}, \ldots, \pi_{k}$ and such that no two tasks of the same rank are scheduled on the same processor. Notice that any permutation of the tasks of the same rank assures the optimality of the schedule with respect to the sum of completion times criterion. This is the family of schedules that we call SPT schedules in the sequel.

We are interested to know whether among the SPT schedules, which are the optimal schedules for the sum of completion times criterion, it is possible to obtain good solutions for the following optimality criteria:

- the maximum sum of completion times per machine: $\max _{1 \leq i \leq m} \sum_{T_{j} \in P_{i}} C_{j}$.

This measure captures the wish of distributing as much as possible the total sum of completion times among the $m$ machines of the system.

- the global fairness [4]: For two vectors $X, Y \in V(\mathcal{I})$, we write $X \preccurlyeq Y$ if $X_{i} \leq Y_{i}$ for all $i$. The global approximation ratio of $X$, denoted by $c_{g b l}(X)$, is the smallest $\alpha$ such that $\vec{X} \preccurlyeq \alpha \vec{Y}$ for all $Y \in V(\mathcal{I})$. Informally $c_{g b l}(X)$ is the smallest $\alpha$ for which $\vec{X}$ is an $\alpha$-approximation, in the coordinate-wise sense, to every vector $Y \in V(\mathcal{I})$. The best global approximation ratio achievable on the instance $\mathcal{I}$ is then defined as

$$
c_{g b l}^{*}(\mathcal{I})=\inf _{X \in V(\mathcal{I})} c_{g b l}(X) .
$$

- the individual fairness: The individual happiness factor compares the completion time of a task with the smallest possible completion time of the same task in any feasible schedule. More formally, we define $c_{i n d}(X)$ to be the smallest $\alpha$ such that $X \preccurlyeq \alpha Y$ for all $Y \in V(\mathcal{I})$. The best approximation ratio achievable on the instance $\mathcal{I}$ is then defined as

$$
c_{\text {ind }}^{*}(\mathcal{I})=\inf _{X \in V(\mathcal{I})} c_{i n d}(X) .
$$

Our approach is in the same vein as the one of Bruno et al. [3] who considered the following question: among all optimal schedules for the sum of 


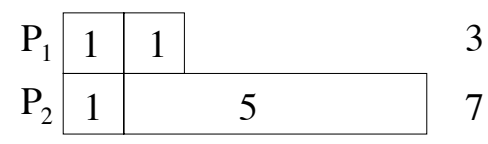

max. sum of completion times $=7$

(total) sum of completion times $=10$

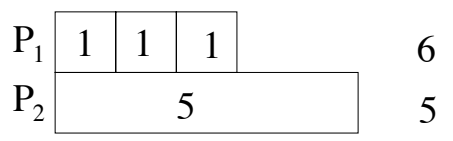

max. sum of completion times $=6$

(total) sum of completion times $=11$

Figure 1. Instance for which the optimal solutions to the problems Minimize $\sum_{j=1}^{n} C_{j}$ and Min MAX SCT are different.

completion times, is it possible to compute one that minimizes the makespan? (They proved that the problem is $\mathcal{N} \mathcal{P}$-hard.) For a related problem, see also [6].

Organization of the paper and our contribution In Section 2, we consider the problem of minimizing the maximum completion time per machine (MIN MAX SCT). We first show that contrarily to the sum of completion times, the problem MIN MAX SCT is $\mathcal{N} \mathcal{P}$-hard. Furthermore, we show that an SPT schedule is a $\left(3-3 / m+1 / m^{2}\right)$-approximation algorithm for the Min MAX SCT problem and that there are instances for which any SPT schedule cannot achieve an approximation guarantee better than $2-\frac{2}{m^{2}+m}$. In Section 3, we consider the global approximation ratio and we prove that SPT schedules have an approximation ratio of $2-1 / m$ (and that no algorithm can have a better approximation ratio if $m=2$ ). Philips et al. [5] presented a 3-approximation for the same problem when release dates are taken into account. For the individual happiness factor however the performance ratio cannot be bounded by any constant but we prove that a SPT schedule obtains the best possible performance guarantee. Finally, we focus on a more restricted version of the individual happiness factor where the obtained solutions are compared with respect to the family of SPT schedules and we prove a 2-approximation bound.

\section{The Min MAX SCT problem}

We first remark that the problem of minimizing the sum of completion times and the MIN MAX SCT problem are different. To see that consider the following instance: three tasks of length 1 and a task of length 5 on two identical processors (see Figure 1). The optimal solution for the problem of minimizing the sum of completion times consists in putting two tasks of length 1 on a processor, and the other two tasks on the other processors (total sum of completion times $=10$, maximum sum of completion times per processor $=7$ ). The optimal solution of MIN MAX SCT consists in putting the three tasks of length 1 on a processor, and the task of length 5 on the other processor (total sum of completion times $=11$, maximum sum of completion time per processor $=6$ ). 


\subsection{Hardness}

We prove in this section that the MIN MAX SCT problem is $\mathcal{N} \mathcal{P}$-hard.

TheOREM 2.1 Min MAX SCT is $\mathcal{N} \mathcal{P}$-hard.

Proof: Consider the decision version of the Min MAX SCT problem: Min MAX SCT problem: Given a system $(\xi, m)$, and a number $k$. Does there exist a schedule such that its maximum sum of completion times is smaller than or equal to $k$ ?

We will show that the PARTITION problem which is known to be $\mathcal{N} \mathcal{P}$-hard [2] can be phrased in terms of problem MIN MAX SCT.

PARTITION problem: Given a collection $C=\left\{x_{1}, x_{2}, \ldots, x_{n}\right\}$ of integers. Does there exist a partition $(A, B)$ of $C$, i.e. $A \cup B=C$ and $A \cap B=\emptyset$, such that $\sum_{x \in A} x=\sum_{x \in B} x$ ?

Given an instance of PARTITION with a collection $C=\left\{x_{1}, x_{2}, \ldots, x_{n}\right\}$ of non decreasing integers $x_{1} \leq x_{2} \leq \cdots \leq x_{n}$, we define a system $(\xi, 2)$ and a limit $k$ such that the MIN MAX SCT instance admits a solution if and only the instance of PARTITION admits a solution. Let $k=\frac{3}{2}\left(\sum_{i=1}^{n} x_{i}\right)-\sum_{i=1}^{n} \frac{x_{i}}{n-i+1}$. We now define the system $(\xi, 2)$ : we have $2 n$ tasks $T_{1}, T_{2}, \ldots, T_{2 n}$ to schedule on 2 processors $P_{1}$ and $P_{2}$. Let $l_{i}$ denote the execution time of task $T_{i}$. Let the execution times $\xi=\left\{l_{1}, l_{2}, \ldots, l_{2 n}\right\}$ be such that $l_{2 j-1}=\sum_{i=1}^{j-1} \frac{x_{i}}{n-i+1}$ and $l_{2 j}=\sum_{i=1}^{j} \frac{x_{i}}{n-i+1}$, for $j \in\{1,2, \ldots, n\}$. For example, (if $n \geq 6$ ), $l_{1}=0, l_{2}=\frac{x_{1}}{n}$,

$$
\begin{aligned}
& l_{3}=\frac{x_{1}}{n}, l_{4}=\frac{x_{1}}{n}+\frac{x_{2}}{n-1}, \\
& l_{5}=\frac{x_{1}}{n}+\frac{x_{2}}{n-1}, l_{6}=\frac{x_{1}}{n}+\frac{x_{2}}{n-1}+\frac{x_{3}}{n-2}, \\
& l_{2 n-1}=\frac{x_{1}}{n}+\frac{x_{2}}{n-1}+\cdots+\frac{x_{n-1}}{2}, \\
& l_{2 n}=\frac{x_{1}}{n}+\frac{x_{2}}{n-1}+\cdots+\frac{x_{n-1}}{2}+x_{n}
\end{aligned}
$$

Let us now show that there is a solution of the MIN MAX SCT problem if and only if there is a solution of PARTITION.

If a partition $(A, B)$ exists for $C$ then there is a solution of the Min MAX SCT problem: we can obtain for our system $(\xi, 2)$ a schedule whose maximum sum of completion times is $\frac{3}{2}\left(\sum_{i=1}^{n} x_{i}\right)-\sum_{i=1}^{n} \frac{x_{i}}{n-i+1}$. This schedule can be obtained by assigning tasks $T_{2 j-1}$ and $T_{2 j}$ at rank $j$ : assign task $T_{2 j}$ to $P_{1}$ and $T_{2 j-1}$ to $P_{2}$ if $x_{j} \in A$, otherwise assign $T_{2 j}$ to $P_{2}$ and $T_{2 j-1}$ to $P_{1}$.

Indeed, the execution time of a task added at the $j^{\text {th }}$ rank will be counted $n-j+1$ times in the sum of the completion times of its processor. For example, the execution time of the last task of a processor will be counted only 
once, whereas the execution time of the first task of a processor will be counted $n$ times (because there are $n$ tasks on each processor). Thus the contribution to the sum of completion times per processor of the task $T_{2 j-1}$ (resp. $T_{2 j}$ ), assigned at the $j^{\text {th }}$ rank, is $(n-j+1) \times l_{2 j-1}$ (resp. $(n-j+1) \times l_{2 j}=$ $\left.(n-j+1) \times\left(l_{2 j-1}+\frac{x_{j}}{n-j+1}\right)\right)$. The difference between these two contributions is then $x_{j}$ : if $x_{j} \in A$ (i.e. $T_{2 j}$ is assigned to $P_{1}$ ) then the contribution of the $j^{\text {th }}$ task of $P_{1}$ is equal to the contribution of the $j^{\text {th }}$ task of $P_{2}$, plus $x_{j}$. Likewise, if $x_{j} \in B$ (i.e. $T_{2 j}$ is assigned to $P_{2}$ ) then the contribution of the $j^{t h}$ task of $P_{2}$ is equal to the contribution of the $j^{\text {th }}$ task of $P_{1}$, plus $x_{j}$.

Thus, the sum of the completion times of the tasks of $P_{1}$ is $\sum_{j=1}^{n} T_{2 j-1}+$ $\sum_{x_{j} \in A} x_{j}$, and the sum of the completion times of the tasks of $P_{2}$ is $\sum_{j=1}^{n} T_{2 j-1}$ $+\sum_{x_{j} \in B} x_{j}$. Moreover $\sum_{j=1}^{n} T_{2 j-1}=(n-1) \frac{x_{1}}{n}+(n-2) \frac{x_{2}}{n-1}+\cdots+\frac{x_{n-1}}{2}=$ $x_{1}-\frac{x_{1}}{n}+x_{2}-\frac{x_{2}}{n-1}+\cdots+x_{n-1}-\frac{x_{n-1}}{2}=\sum_{i=1}^{n} x_{i}-\sum_{i=1}^{n} \frac{x_{i}}{n-i+1}$. So the sum of the completion times of the tasks of $P_{1}$ is $\left(\sum_{i=1}^{n} x_{i}-\sum_{i=1}^{n} \frac{x_{i}}{n-i+1}\right)+$ $\sum_{x_{j} \in A} x_{j}$, and the sum of the completion times of the tasks of $P_{2}$ is $\left(\sum_{i=1}^{n} x_{i}-\right.$ $\left.\sum_{i=1}^{n} \frac{x_{i}}{n-i+1}\right)+\sum_{x_{j} \in B} x_{j}$.

If there is partition of $C$ (i.e. $\sum_{x_{j} \in A}=\sum_{x_{j} \in B}=\frac{\sum_{i=1}^{n} x_{i}}{2}$ ) then the maximum sum of completion times per processor is equal to $\left(\sum_{i=1}^{n} x_{i}-\sum_{i=1}^{n} \frac{x_{i}}{n-i+1}\right)+$ $\frac{\sum_{i=1}^{n} x_{i}}{2}=\frac{3}{2}\left(\sum_{i=1}^{n} x_{i}\right)-\sum_{i=1}^{n} \frac{x_{i}}{n-i+1}=k$. Thus, if there is a solution of PARTITION, there is a solution of the MIN MAX SCT problem.

Let us now show that if there is a solution of the Min MAX SCT problem, then there is also a solution of PARTITION. If the maximum sum of the completion times per processor is smaller than or equal to $\frac{3}{2}\left(\sum_{i=1}^{n} x_{i}\right)-\sum_{i=1}^{n} \frac{x_{i}}{n-i+1}$, then we have a SPT schedule. Indeed, the total sum of the completion times of a SPT schedule is $S=2 \times\left(\frac{3}{2}\left(\sum_{i=1}^{n} x_{i}\right)-\sum_{i=1}^{n} \frac{x_{i}}{n-i+1}\right)$, as we saw it above, and a schedule has a minimum total sum of completion times if and only if it is a SPT schedule [3]. Since the total sum of completion times cannot be greater than twice the maximum sum of completion times per processor, a schedule which is a solution of the MIN MAX SCT problem is a SPT schedule. In any SPT schedule, the tasks of length $l_{2 i}$ and $l_{2 i-1}$ are at the $i^{\text {th }}$ rank, because $l_{1}<l_{2} \leq l_{3}<\ldots l_{2 j-1}<l_{2 j} \leq l_{2 j+1}<l_{2 j+2} \leq \cdots<l_{n}$. So, in any SPT schedule, the sum of completion times of $P_{1}$ minus the one of $P_{2}$ is equal to $\sum_{T_{2 j} \in P_{1}} x_{j}-\sum_{T_{2 j} \in P_{2}} x_{j}$. If there is a solution of the Min MAX SCT problem with $k=\frac{3}{2}\left(\sum_{i=1}^{n} x_{i}\right)-\sum_{i=1}^{n} \frac{x_{i}}{n-i+1}$ then the sum of the completion times of $P_{1}$ is equal to the sum of the completion times of $P_{2}$ (otherwise the sum of completion times of all the tasks would be smaller than $S$ ), and then $\sum_{T_{2 j} \in P_{1}} x_{j}=\sum_{T_{2 j} \in P_{2}} x_{j}$ : there is a partition of $C$ and we can construct this partition $(A, B)$ by placing $x_{j}$ in $A$ if $T_{2 j}$ is assigned to $P_{1}$ and $x_{j}$ in $B$ if $T_{2 j}$ 
is assigned to $P_{2}$.

\subsection{Approximation}

In this section, we show that an optimal algorithm for the sum of completion times criterion gives a 3-approximate solution for the MIN MAX SCT problem. Consider the following algorithm, denoted by $\mathrm{SPT}_{\text {greedy }}$ :

Order tasks by non decreasing execution times. At each step $i$, for $1 \leq i \leq n$, schedule the current task on the processor which has the smallest completion time.

This algorithm gives an optimal solution for the problem of minimizing the sum of completion times of the tasks. Let us show that this algorithm is a $\left(3-\frac{3}{m}+\frac{1}{m^{2}}\right)$-approximation algorithm for MIN MAX SCT.

In order to let $\mathrm{SPT}_{\text {greedy }}$ be deterministic when several processors have the smallest execution time, we will refer in the proofs to the following algorithm, which is a greedy SPT algorithm (Proposition 2.1 shows that we add at each step a task on a processor which has the smallest completion time):

Order tasks by non decreasing execution times. At each step $i$, for $1 \leq i \leq n$, schedule the current task on the processor $P_{i \bmod m}$.

\section{Proposition 2.1}

a) At the beginning of step $i$ of $S P T_{\text {greedy }}$, the processor $P_{i \bmod m}$ is the processor which has the smallest completion time and the smallest sum of completion times.

b) At the end of step $i$ of $S P T_{\text {greedy }}$, the processor $P_{i \bmod m}$ is the processor which has the largest sum of completion times.

\section{Proof:}

a) We are at the beginning of step $i$. Let $c$ be the processor $P_{i \bmod m}$, and let $p$ denote its completion time. Let us show that $c$ has the smallest completion time:

- For each $k>i \bmod m$, processor $P_{k}$ has a completion time greater than or equal to $p$. Indeed $P_{k}$ has the same number of tasks as $c$ and, for each $i$ such that $1 \leq i \leq$ number of tasks on $c$, the $i$-th task of $P_{k}$ is greater than or equal to the $i$-th task of $c$, by construction. 
- For each $k<i \bmod m$, processor $P_{k}$ has a completion time greater than or equal to $p$. Indeed the number of tasks on $P_{k}$ is equal to the number of tasks on $c$ plus one, and, for each $i$ such that $1 \leq i \leq$ (number of tasks on $c$ ), the $(i+1)$-th task of $P_{k}$ is greater than or equal to the $i$-th task of $c$, by construction.

We use the same reasoning to show that $c$ has the smallest sum of completion times.

b) We are at the end of step $i$. Let $c$ be the processor $P_{i \bmod m}$, and let $p$ denote its sum of completion times at this step. Let us show that $c$ has the largest sum of completion times:

- For each $k>i \bmod m$, processor $P_{k}$ has a sum of completion times smaller than or equal to $p$. Indeed $P_{k}$ has one task less than $c$ and, for each $i$ such that $1 \leq i<$ number of tasks on $c$, the $i$-th task of $P_{k}$ is smaller than or equal to the $(i+1)$-th task of $c$, by construction.

- For each $k<i \bmod m$, processor $P_{k}$ has a sum of completion times smaller or equal to $p$. Indeed $P_{k}$ has the same number of tasks as $c$ and, for each $i$ such that $1 \leq i \leq$ number of tasks on $c$, the $i$-th task of $P_{k}$ is smaller than or equal to the $i$-th task of $c$, by construction.

Proposition 2.2 Let OPT be the maximum sum of completion times of a solution of MIN MAX SCT. We have:

$$
O P T \geq \frac{\operatorname{Min}\left(\sum_{j=1}^{n} C_{j}\right)}{m},
$$

where $\operatorname{Min}\left(\sum_{j=1}^{n} C_{j}\right)$ is the optimal value for the problem of minimizing the sum of completion times.

Proof: We will prove this proposition by contradiction. Let us suppose that we have $O P T<\frac{\operatorname{Min}\left(\sum_{j=1}^{n} C_{j}\right)}{m}$.

By definition each processor has a sum of completion times smaller than or equal to OPT: $\forall i \in\{1, \ldots, m\}, \sum_{T_{j} \in P_{i}} C_{j} \leq O P T$.

So, $\sum_{j=1}^{n} C_{j}=\sum_{i=1}^{m} \sum_{T_{j} \in P_{i}} C_{j} \leq m \times O P T$, and $O P T \geq \frac{\sum_{j=1}^{n} C_{j}}{m} \geq$ $\frac{\operatorname{Min}\left(\sum_{j=1}^{n} C_{j}\right)}{m}$, a contradiction. 
THEOREM 2.2 The algorithm SPT greedy achieves an approximation guarantee of $\left(3-\frac{3}{m}+\frac{1}{m^{2}}\right)$ for MiN MAX SCT.

Proof: Before we add the last task $T_{n}$, the processor $P_{x}$ on which $T_{n}$ will be scheduled has the smallest sum of completion times, denoted by $p$, and the smallest completion time, denoted by $e$ (Proposition 2.1 a). Let $l_{n}$ denote the execution time of task $T_{n}$. The processor on which the last task is scheduled has the largest sum of completion times (Proposition $2.1 \mathrm{~b}$ ), so:

$$
\begin{aligned}
\max _{1 \leq i \leq m} \sum_{T_{j} \in P_{i}} C_{j} & =\sum_{T_{j} \in P_{x}} C_{j}=p+e+l_{n} \\
& \leq \frac{\sum_{j=1}^{n-1} C_{j}}{m}+e+l_{n}
\end{aligned}
$$

Since $\mathrm{SPT}_{\text {greedy }}$ gives an optimal solution of the minimum sum of completion times problem, and since the completion time of $T_{n}$ in $\mathrm{SPT}_{\text {greedy }}$ is $e+l_{n}$, we have:

$$
\begin{aligned}
\max _{1 \leq i \leq m} \sum_{T_{j} \in P_{i}} C_{j} & \leq \frac{\operatorname{Min}\left(\sum_{j=1}^{n} C_{j}\right)-\left(e+l_{n}\right)}{m}+e+l_{n} \\
& \leq \frac{\operatorname{Min} \sum_{j=1}^{n} C_{j}}{m}+\left(1-\frac{1}{m}\right)\left(e+l_{n}\right) .
\end{aligned}
$$

Since $e+\frac{l_{n}}{m} \leq O P T$ (because $e$ is the minimum completion time at step $n-1$ ), and $l_{n} \leq O P T$, we have:

$$
\begin{aligned}
e+l_{n} & =e+\frac{l_{n}}{m}+\frac{(m-1) l_{n}}{m} \\
& \leq\left(1+\frac{m-1}{m}\right) O P T \\
& \leq\left(2-\frac{1}{m}\right) O P T
\end{aligned}
$$

Moreover, since $\frac{\operatorname{Min} \sum_{j=1}^{n} C_{j}}{m} \leq O P T$ (Proposition 2.2), we have:

$$
\begin{aligned}
\max _{1 \leq i \leq m} \sum_{T_{j} \in P_{i}} C_{j} & \leq O P T+\left(1-\frac{1}{m}\right)\left(2-\frac{1}{m}\right) O P T \\
& \leq\left(3-\frac{3}{m}+\frac{1}{m^{2}}\right) O P T .
\end{aligned}
$$

Lower bound for SPT greedy $_{\text {. We now show that SPT }}$ greedy does not achieve an approximation guarantee better than $2-\frac{2}{m^{2}+m}$. Consider the following instance: $m$ processors, $m \times(m-1)$ tasks of length 1 and a task of length $B=\sum_{i=1}^{m} i=\frac{m(m+1)}{2}$.

$\mathrm{SPT}_{\text {greedy }}$ will schedule $m-1$ tasks of length 1 on each processor, and the task of length $B$ will be the last task of the first processor (see Figure 2). The 


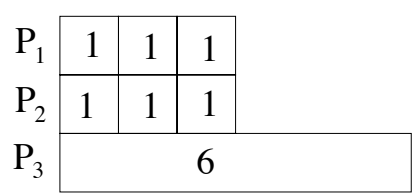

max. sum of completion times $=6$

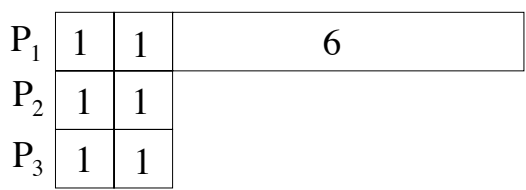

max. sum of completion times $=11$

Figure 2. $\mathrm{SPT}_{\text {greedy }}$ does not achieve an approximation guarantee better than $\left(2-\frac{2}{m^{2}+m}\right)$ : example with $\mathrm{m}=3$

maximum sum of completion times is then $\sum_{i=1}^{m-1} i+(m-1)+B$, which is equal to $2\left(\sum_{i=1}^{m} i\right)-1$.

An optimal solution for MIN MAX SCT would be the following one: schedule $m$ tasks of length 1 on each of the $(m-1)$ first processors, and the task of length $B$ on the last processor. The maximum sum of completion times in this solution is then $\sum_{i=1}^{m} i$.

So the ratio between the maximum sum of completion times of these two schedules is $\frac{2\left(\sum_{i=1}^{m} i\right)-1}{\sum_{i=1}^{m} i}$, which is equal to $2\left(1-\frac{1}{m^{2}+m}\right)$, which tends towards 2 when $m$ gets large.

\section{Fairness measures}

In this section we will consider fairness measures in order to compare a schedule given by the greedy SPT algorithm, $\mathrm{SPT}_{\text {greedy }}$, to any other schedule.

\subsection{Global fairness measure}

We will first use the fairness measure introduced in [4], namely the global approximation ratio. We prove that $\mathrm{SPT}_{\text {greedy }}$ has a global approximation ratio of $2-\frac{1}{m}$ for the problem that we consider (and which is denoted by P $\|$ all ), and that no algorithm can achieve a better global approximation ratio if $m=2$.

THEOREM 3.1 One has $c_{g b l}^{*}(\mathcal{I}) \leq 2-\frac{1}{m}$ for all instances $\mathcal{I}$ of scheduling on $m$ identical parallel machines ( $P \|$ all), for any $m \geq 1$. Moreover the completion times vector $X$ of a schedule returned by $S P T_{\text {greedy }}$ verifies $c_{g b l}(X) \leq 2-\frac{1}{m}$.

Proof: Let us consider an instance $\mathcal{I}$ of tasks $T_{i}(i \in\{1, \ldots, n\})$ ordered by increasing lengths. Let MR be the maximal ratio between a schedule $X_{S P T}$ returned by $\mathrm{SPT}_{\text {greedy }}$ and any schedule $Y: \mathrm{MR}=\max _{Y} \frac{\overrightarrow{X_{S P T}}}{\vec{Y}}$, where $\frac{X}{Y}$ 
means $\max _{i} \frac{X_{i}}{Y_{i}}$. So we have $\forall Y \in V(\mathcal{I}), \frac{\overrightarrow{X_{S P T}}}{\bar{Y}} \leq \mathrm{MR}$, and then $\overrightarrow{X_{S P T}} \preccurlyeq$ MR $\vec{Y}$. So MR $=c_{g b l}\left(X_{S P T}\right)$. We will show that MR cannot be greater than $2-\frac{1}{m}$. Let us consider that this ratio is the $\imath^{\text {th }}$ coordinate of $\overrightarrow{X_{S P T}}$, divided by the $i^{\text {th }}$ coordinate of $\vec{Y}(i \in\{1, \ldots, n\})$.

The worst ratio can be achieved if the completion time of the $i^{\text {th }}$ task is as large as possible in the $\mathrm{SPT}_{\text {greedy }}$ schedule. By construction, in a schedule returned by $\mathrm{SPT}_{\text {greedy }}$, the $i^{\text {th }}$ completion time is the completion time of the task $T_{i}$, and $T_{i}$ is started after the tasks from $T_{1}$ to $T_{i-1}$, and before the tasks from $T_{i+1}$ to $T_{n}$. Since $\mathrm{SPT}_{\text {greedy }}$ is a greedy algorithm, the worst completion time of the $i^{\text {th }}$ task is achieved when the $(i-1)$ first tasks are completed when the $i^{\text {th }}$ task start to be executed: in this case, the completion time of $T_{i}$ is $\frac{\sum_{j=1}^{i-1} l_{j}}{m}+l_{i}$. So the worst completion time of $T_{i}$ in an $\mathrm{SPT}_{\text {greedy }}$ schedule is $\frac{\sum_{j=1}^{i-1} l_{j}}{m}+l_{i}$.

Let us now find the minimal value which can be taken by the $i^{\text {th }}$ coordinate of $\vec{Y}$, an ordered completion time vector of a schedule of $\mathcal{I}$. Note that this value cannot be smaller than $l_{i}$ : indeed $\vec{Y}_{i}$ is the $i^{\text {th }}$ completion time and is then greater or equal to $(i-1)$ other completion times, and $l_{i}$ is the length of the $i^{\text {th }}$ smallest task. Note also that $\vec{Y}_{i}$ cannot be smaller than $\frac{\sum_{j=1}^{i} l_{j}}{m}$ : indeed this is the minimum completion time of the $i$ smallest tasks (when no processor is idle). Therefore, we have:

$$
\mathrm{MR} \leq \frac{\frac{\sum_{j=1}^{i-1} l_{j}}{m}+l_{i}}{\max \left(\frac{\sum_{j=1}^{i} l_{j}}{m}, l_{i}\right)}
$$

Let $A$ denote $\frac{\sum_{j=1}^{i} l_{j}}{m}$. We now consider the two possible cases:

- Suppose that $l_{i} \geq A$, we have: $\mathrm{MR} \leq \frac{\frac{\sum_{j=1}^{i-1} l_{j}}{m}+l_{i}}{l_{i}} \leq \frac{A-\frac{l_{i}}{m}+l_{i}}{l_{i}} \leq \frac{A}{l_{i}}+1-$ $\frac{1}{m} \leq 2-\frac{1}{m}$

- Suppose that $l_{i}<A$, we have: $\mathrm{MR} \leq \frac{\frac{\sum_{j=1}^{i-1} l_{j}}{m}+l_{i}}{\frac{\sum_{j=1}^{i} l_{j}}{m}} \leq \frac{A-\frac{l_{i}}{m}+l_{i}}{A} \leq 1+$ $\frac{l_{i}}{A}\left(1-\frac{1}{m}\right) \leq 2-\frac{1}{m}$

In both cases, we have MR $\leq 2-\frac{1}{m}$. Since MR $=c_{g b l}\left(X_{S P T}\right)$, and since $X_{S P T}$ is the completion time vector of the $\mathrm{SPT}_{\text {greedy }}$ schedule of $\mathcal{I}$, we proved that a schedule $X$ returned by $\mathrm{SPT}_{\text {greedy }}$ verifies $c_{g b l}(X) \leq 2-\frac{1}{m}$, and so that $c_{g b l}^{*}(\mathcal{I}) \leq 2-\frac{1}{m}$. 


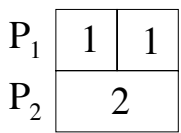

completion times vector: $\mathrm{X} 1=(1,2,2)$

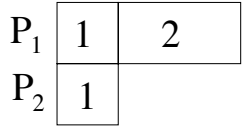

completion times vector: $\mathrm{X} 2=(1,1,3)$

Figure 3. Example where we have $c_{g b l}^{*}(\mathcal{I})=\frac{3}{2}=2-\frac{1}{m}$.

Let us now show that it is not possible to have $c_{g b l}^{*}(\mathcal{I})<2-\frac{1}{m}$ for all instances $\mathcal{I}$ of (P $\|$ all). Indeed, if $m=2$, it is not possible to have always $c_{\text {glob }}^{*}(\mathcal{I})<\frac{3}{2}$ :

THEOREM 3.2 It is not possible to have $c_{g b l}^{*}(\mathcal{I})<\frac{3}{2}$ for all instances $\mathcal{I}$ of scheduling on two identical parallel machines.

Proof: let us consider a system with two processors, $P_{1}$ and $P_{2}$. Let us consider the following instance $\mathcal{I}$ : a task $T_{1}$ of length $1, T_{2}$ of length 1 , and $T_{3}$ of length 2 . Consider the two completion times vector $X_{1}=(1,2,2)$ (obtained by putting $T_{1}$ and $T_{2}$ on $P_{1}$ and $T_{3}$ on $P_{2}$ : see Figure 3$)$ and $X_{2}=(1,1,3)$ (obtained by putting $T_{1}$ and $T_{3}$ on $P_{1}$ and $T_{2}$ on $P_{2}$ ). We have: $c_{g b l}\left(X_{1}\right)=2$, and $c_{g b l}\left(X_{2}\right)=\frac{3}{2}$. For each vector $Y \in V(\mathcal{I})$, we have $\overrightarrow{X_{1}} \preccurlyeq \vec{Y}$ or $\overrightarrow{X_{2}} \preccurlyeq \vec{Y}$. So for each vector $Y \in V(\mathcal{I})$, we have $c_{g b l}(Y) \geq \frac{3}{2}$, and so $c_{g b l}^{*}(\mathcal{I})=\frac{3}{2}$.

\subsection{Individual fairness measure}

In this section, we will compare the completion time of each task in a solution given by $\mathrm{SPT}_{\text {greedy }}$ to the best completion time this task could have in another schedule.

THEOREM $3.3 c_{\text {ind }}^{*}(\mathcal{I}) \leq 1+\frac{n-1}{m}$ for all instances $\mathcal{I}$ of scheduling on $m$ identical parallel machines ( $P \|$ ind_all), for any $m \geq 1$. Moreover the completion times vector $X$ of a schedule returned by $S P T_{\text {greedy }}$ verifies $c_{\text {ind }}(X) \leq 1+\frac{n-1}{m}$.

Proof: Let us consider an instance $\mathcal{I}$ of tasks $T_{i}(i \in\{1, \ldots, n\})$ ordered by non decreasing lengths. It is easy to see that $c_{i n d}\left(X_{S P T}\right)$ is the maximal ratio between a schedule $X_{S P T}$ returned by $\mathrm{SPT}_{\text {greedy }}$ and any schedule $Y$. We will show that $c_{i n d}\left(X_{S P T}\right)$ cannot be greater than $1+\frac{n-1}{m}$. Let us consider that this ratio is the $i^{\text {th }}$ coordinate of $X_{S P T}$, divided by the $i^{\text {th }}$ coordinate of $Y$ $(i \in\{1, \ldots, n\})$.

The worst ratio can be reached if the completion time of $T_{i}$ is as large as possible in the $\mathrm{SPT}_{\text {greedy }}$ schedule. Since $\mathrm{SPT}_{\text {greedy }}$ is a greedy algorithm, this 
is achieved when the $(i-1)$ first tasks are completed when $T_{i}$ start to be executed: in this case, the completion time of $T_{i}$ is $\frac{\sum_{j=1}^{i-1} l_{j}}{m}+l_{i}$. So the maximal value which can be taken by the $\imath^{\text {th }}$ coordinate of $X_{S P T}$ is $\frac{\sum_{j=1}^{i-1} l_{j}}{m}+l_{i}$. The minimal value which can be taken by the $i^{\text {th }}$ coordinate of $Y$ is $l_{i}$ : this is achieved in a schedule in which $T_{i}$ is in the first position. We have:

$c_{i n d}\left(X_{S P T}\right) \leq \frac{\frac{\sum_{j=1}^{i-1} l_{j}}{m}+l_{i}}{l_{i}} \leq 1+\frac{\sum_{j=1}^{i-1} l_{j}}{m l_{i}} \leq 1+\frac{(i-1) l_{i}}{m l_{i}} \leq 1+\frac{n-1}{m}$.

Since $c_{\text {ind }}\left(X_{S P T}\right) \leq 1+\frac{n-1}{m}$, we have $c_{\text {ind }}^{*}(\mathcal{I}) \leq 1+\frac{n-1}{m}$.

THEOREM 3.4 It is not possible to have $c_{\text {ind }}^{*}(\mathcal{I})<1+\frac{n-1}{m}$ for all instances $\mathcal{I}$ of scheduling on $m$ identical parallel machines $(P \|$ ind_all $)$.

Proof: Let us consider a system with $m$ processors and the following instance $\mathcal{I}: m+1$ tasks of length 1 . We have $c_{i n d}^{*}(\mathcal{I})=2$ because at least one of the tasks will have a completion time of 2 . Since $c_{i n d}^{*}(\mathcal{I})=2=1+\frac{n-1}{m}$, it is not possible to have $c_{\text {ind }}^{*}(\mathcal{I})<1+\frac{n-1}{m}$ for all instances $\mathcal{I}$.

\subsection{Individual fairness measure among SPT schedules}

In this section, we will compare the completion time of each task in an $\mathrm{SPT}_{\text {greedy }}$ schedule to the best completion time this task could have in another SPT schedule.

In addition to the notations introduced in the last sections, we define $V_{S P T}$ as the set of all completion times vectors that are induced by some SPT solutions of the instance. We also define $c_{S P T}(X)$ to be the smallest $\alpha$ such that $X \preccurlyeq \alpha Y$ for all $Y \in V_{S P T}(\mathcal{I})$. This can be informally viewed as the global approximation ratio of $X$ : it is the smallest $\alpha$ for which $X$ is an $\alpha$ approximation to every vector $Y \in V_{S P T}(\mathcal{I})$. The best approximation ratio achievable on the instance $\mathcal{I}$ is then defined as

$$
c_{S P T}^{*}(\mathcal{I})=\inf _{X \in V_{S P T}(\mathcal{I})} c_{S P T}(X) .
$$

THEOREM $3.5 c_{S P T}^{*}(\mathcal{I}) \leq 2$ for all instances $\mathcal{I}$ of scheduling on $m$ identical parallel machines $\left(P \|\right.$ ind_all $\left.{ }_{S P T}\right)$. Moreover any $S P T$ schedule $X$ verifies $c_{S P T}(X) \leq 2$.

Proof: Let us consider an instance $\mathcal{I}$ of tasks $T_{i}(i \in\{1, \ldots, n\})$ ordered by increasing lengths. 


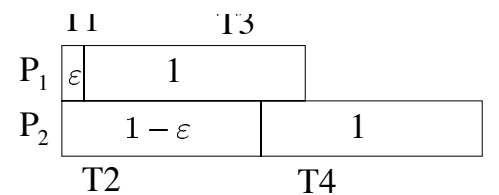

completion times vector: $(\varepsilon, 1-\varepsilon, \varepsilon+1,2-\varepsilon)$

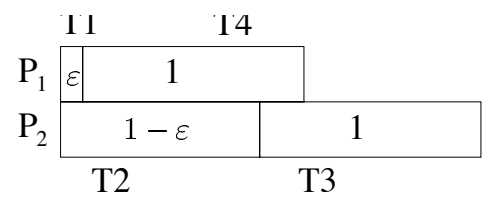

completion times vector: $(\varepsilon, 1-\varepsilon, 2-\varepsilon, \varepsilon+1)$

Figure 4. Example where $c_{S P T}^{*}(\mathcal{I})$ tends towards 2 when $\varepsilon$ tends towards 0 .

It is easy to see that $c_{S P T}(X)$ is the maximal ratio between a SPT schedule $X$ and any SPT schedule $Y$. We will show that $c_{S P T}(X)$ cannot be greater than 2. Let us consider that this ratio is the $\imath^{\text {th }}$ coordinate of $X$, divided by the $i^{\text {th }}$ coordinate of $Y(i \in\{1, \ldots, n\})$. Let $P_{i X}$ (resp. $P_{i Y}$ ) be the processor on which $T_{i}$ is scheduled in the solution $X$ (resp $Y$ ), and let $r_{i}$ denote the rank on which the task $T_{i}$ is scheduled.

The worst ratio is reached if the completion time of $T_{i}$ is as large as possible in the schedule $X$. This is achieved when the tasks before $T_{i}$ on $P_{i X}$ are as large as possible: each largest task on each rank smaller than $r_{i}$ is on $P_{i X}$ in the solution $X$. Let $B_{m}$ denote the execution time of these tasks. $B_{m}$ is the execution time of the tasks on the processor $P_{m}$ in an $\mathrm{SPT}_{\text {greedy }}$ schedule. In the same way, the worst ratio is reached if the completion time of $T_{i}$ is as small as possible in the schedule $Y$ : each smallest task on each rank smaller than $r_{i}$ is on $P_{i Y}$ in the solution $Y$. Let $B_{1}$ denote the execution time of these tasks. $B_{1}$ is the execution time of the tasks on the processor $P_{1}$ in an $\mathrm{SPT}_{\text {greedy }}$ schedule. So the difference between the completion time of $T_{i}$ in $X$ and in $Y$ is equal to $\delta=B_{m}-B_{1}$. This number is smaller than or equal to the length of each task in the rank $r_{i}$, and then is smaller than or equal to $l_{i}$, the length of $T_{i}$ (this property is a direct consequence of Proposition 2.1). We have:

$$
c_{S P T}(X) \leq \frac{B_{m}+l_{i}}{B_{1}+l_{i}} \leq \frac{B_{1}+\delta+l_{i}}{B_{1}+l_{i}} \leq 2 .
$$

For any SPT schedule $X$ of an instance $\mathcal{I}$, we have: $c_{S P T}(X) \leq 2$, and so $c_{S P T}^{*}(\mathcal{I}) \leq 2$.

Let us now show that this bound is the best possible.

THEOREM 3.6 Let $\varepsilon$ be any small number such that $\varepsilon>0$. It is not possible to have $c_{S P T}^{*}(\mathcal{I})<2-\varepsilon$ for all instances $\mathcal{I}$ of scheduling on $m$ identical parallel machines $(P \|$ ind_all $S P T)$.

Proof: For the ease of presentation we give a proof for a system with two processors. Let us consider the following instance $\mathcal{I}$ : a task $T_{1}$ of length $\varepsilon, T_{2}$ of 
length $1-\varepsilon$, and two tasks $T_{3}$ and $T_{4}$ of length 1 . Assume that $\varepsilon<1$. The completion times vectors corresponding to the two possible SPT schedules are $X_{1}=(\varepsilon, 1-\varepsilon, 1+\varepsilon, 2-\varepsilon)$ and $X_{2}=(\varepsilon, 1-\varepsilon, 2-\varepsilon, 1+\varepsilon)$ (see Figure 4). We have: $c_{S P T}^{*}(\mathcal{I})=c_{S P T}\left(X_{1}\right)=c_{S P T}\left(X_{2}\right)=\frac{2-\varepsilon}{1+\varepsilon}<2-\varepsilon$, which tends towards 2 when $\varepsilon$ tends towards 0 . If $m>2$, the proof is the same except that we add $m-2$ tasks of length $1-\varepsilon$ and $m-2$ tasks of length 1 to the instance described for the case where $m=2$.

\section{References}

[1] R.W. Conway, W.L. Maxwell, and L.W. Miller, Theory of scheduling. Addison-Wesley (1967).

[2] M. Garey and D. Johnson, Computers and Intractability: A Guide to the Theory of NPCompleteness. W H Freeman \& Co.

[3] J. Bruno, E.G. Coffman Jr., and R. Sethi, Algorithms for minimizing mean flow time. Proceedings of IFIP Congress 74, 504-510, Stockholm, Sweden, August 5-10, 1974, NorthHolland, (1974).

[4] A. Kumar and J. Kleinberg, Fairness Measures for Resource Allocation. Proceedings of 41st IEEE Symposium on Foundations of Computer Science, 75-85 (2000).

[5] C. Phillips, C. Stein and J. Wein, Scheduling jobs that arrive over time. Proceedings of the Fourth Workshop on Algorithms and Data Structures, LNCS 955, Springer, 86-97 (1995).

[6] C. Stein and J. Wein, On the existence of schedules that are near-optimal for both makespan and total weighted completion time. Operations Research Letters 21 (3), 115122 (1997). 